\title{
THE EFFECT OF PERTURBATIONS OF OPERATOR-VALUED FRAME SEQUENCES AND FUSION FRAMES ON THEIR DUALS
}

\author{
GitTa Kutyniok, Victoria Paternostro And Friedrich PhilipP
}

\begin{abstract}
Fusion frames, and, more generally, operator-valued frame sequences are generalizations of classical frames, which are today a standard notion when redundant, yet stable sequences are required. However, the question of stability of duals with respect to perturbations has not been satisfactorily answered. In this paper, we quantitatively measure this stability by considering the associated deviations of the canonical and alternate dual sequences from the original ones. It is proven that operator-valued frame sequences are indeed stable in this sense. Along the way, we also generalize existing definitions for fusion frame duals to the infinite-dimensional situation and analyze how they perform with respect to a list of desiderata which, to our minds, a fusion frame dual should satisfy. Finally, we prove a similar stability result as above for fusion frames and their canonical duals.
\end{abstract}

Mathematics subject classification (2010): Primary 42C15; Secondary 46C05.

Keywords and phrases: Operator-valued frame, frame sequence, fusion frame, perturbation, dual frame, fusion frame dual.

\section{REFERENCES}

[1] A. A. Arefijamanl And S. Ghasemi, On characterization and stability of alternate dual of $g$ frames, Turk. J. Math. 37 (2013), 71-79.

[2] R. BALAn, Stability theorems for Fourier frames and wavelet Riesz bases, J. Fourier Anal. Appl. 3 (1997), 499-504.

[3] S. BISHOP, C. HeIL, Y. Y. Koo, AND J. K. Lim, Invariances of frame sequences under perturbations, Linear Algebra Appl. 432 (2010), 1501-1514.

[4] P. G. CASAZZA AND O. Christensen, Perturbation of operators and applications to frame theory, J. Fourier Anal. Appl. 3 (1997), 543-557.

[5] P. G. CASAzZA And G. Kutyniok, Frames of subspaces, Wavelets, Frames and Operator Theory (College Park, MD, 2003), Contemp. Math. 345, Amer. Math. Soc., Providence, RI, 2004, 87-113.

[6] P. G. CASAzZA, G. KUTYNiok, And S. Li, Fusion frames and distributed processing, Appl. Comput. Harmon. Anal. 25 (2008), 114-132.

[7] P. G. CasazZA, G. LiU, C. ZhaO, AND P. ZhaO, Perturbations and irregular sampling theorems for frames, IEEE Transactions on Information Theory 52 (2006), 4643-4648.

[8] P. G. CasazZa And J. C. TRemain, The Kadison-Singer problem in mathematics and engineering, Proc. Natl. Acad. Sci. 103 (2006), 2032-2039.

[9] O. Christensen, An introduction to frames and Riesz bases, Birkhäuser, Boston, Basel, Berlin 2003.

[10] O. Christensen AND Y. C. Eldar, Oblique dual frames and shift-invariant spaces, Appl. Comput. Harmon. Anal. 17 (2004), 48-68.

[11] O. Christensen And C. Heil, Perturbations of Banach frames and atomic decompositions, Math. Nachr. 185 (1997), 33-47.

[12] O. Christensen, H. O. Kim, R. Y. Kim, And J. K. Lim, Perturbation of frame sequences in shift-invariant spaces, J. Geom. Anal. 15 (2005), 181-192.

[13] R. Duffin And A. Schaeffer, A class of nonharmonic Fourier series, Trans. Amer. Math. Soc. 72 (1952), 341-366. 
[14] Y. C. Eldar And G. D. Forney, Optimal tight frames and quantum measurement, IEEE Trans. Inform. Theory 48 (2002), 599-610.

[15] H. G. Feichtinger AND W. SUN, Stability of Gabor frames with arbitrary sampling points, Acta Mathematica Hungarica 113 (2006), 187-212.

[16] P. GĂvruţA, On the duality of fusion frames, J. Math. Anal. Appl. 333 (2007), 871-879.

[17] C. Heil, Y. Y. Koo, AND J. K. Lim, Duals of frame sequences, Acta Appl. Math 107 (2009), 75-90.

[18] S. Heineken, E. Matusiak, And V. Paternostro, Perturbed frame sequences: Canonical dual systems, approximate reconstructions and applications, Int. J. Wavelets Multiresolut. Inf. Process. 12 (2014), 1450019-1-1450019-19.

[19] S. Heineken And P. M. Morillas, Properties of finite dual fusion frames, Linear Algebra Appl. 453 (2014), 1-27.

[20] S. Heineken, P. M. Morillas, A. M. Benavente, and M. I. Zakowicz, Dual fusion frames, Arch. Math. 103 (2014), 355-365.

[21] A. A. Hemmat AND J.-P. Gabardo, The uniqueness of shift-generated duals for frames in shiftinvariant subspaces, J. Fourier Anal. Appl. 13 (2007), 589-606.

[22] V. Kaftal, D. R. Larson, And S. ZHAng, Operator-valued frames, Trans. Amer. Math. Soc. 361 (2009), 6349-6385.

[23] T. Kato, Perturbation theory for linear operators, Second Edition, Springer, Berlin, Heidelberg, New York, 1976.

[24] G. Kutyniok, J. Lemvig, AND W.-Q. Lim, Optimally sparse approximations of 3D functions by compactly supported shearlet frames, SIAM J. Math. Anal. 44 (2012), 2962-3017.

[25] G. Kutyniok, V. Mehrmann, And P. Petersen, Regularization and numerical solution of the inverse scattering problem using shearlet frames, J. Inverse Ill-Posed Probl., to appear.

[26] S. LI, On general frame decompositions, Numer. Funct. Anal. Optim. 16 (1995), 1181-1191.

[27] A. S. MARKUS, On some properties of linear operators connected with the notion of gap (Russian), Kishinev Gos. Univ. Uchen. Zap. 39 (1959), 265-272.

[28] L. Oeding, E. Robeva, And B. Sturmfels, Decomposing tensors into frames, Adv. Appl. Math. 73 (2016), 125-153.

[29] F. Riesz And B. Sz.-Nagy, Functional analysis, Translated by Leo F. Boron. Frederick Ungar Publishing Co., New York, 1955.

[30] T. STROHMER AND R. W. HEATH, Grassmannian frames with applications to coding and communication, Appl. Comput. Harmon. Anal. 11 (2003), 243-262.

[31] W. Sun, g-frames and g-Riesz bases, J. Math. Anal. Appl. 322 (2006), 437-452.

[32] W. Sun, Stability of g-frames, J. Math. Anal. Appl. 326 (2007), 858-868.

[33] P. ZhaO, C. ZHAO, AND P. G. CASAZZA, Perturbation of regular sampling in shift-invariant spaces for frames, IEEE Trans. Inf. Theory 52 (2006), 4643-4648. 